\title{
OPTIMAL PRICE DECREMENTAL STRATEGY FOR DUTCH AUCTIONS*
}

\author{
WING HO YUEN ${ }^{\dagger}$, CHI WAN SUNG ${ }^{\ddagger}$, AND WING SHING WONG ${ }^{\S}$
}

\begin{abstract}
In a Dutch auction, the price of an item decreases incrementally from the starting price at regular intervals. A bidder may buy the item at any time and stop the auction at the current price. This paper presents an optimal price decrement strategy in a Dutch auction, such that the expected revenue of the auction host is maximized. Properties of the optimal solution and a simple iterative solution methodology are discussed. Numerical studies show that significant gain could be obtained compared with a simple reference strategy.
\end{abstract}

Subject terms. Dutch auction; optimal auction; bidding; 3G; wireless application protocol; optimization; time discounting; online auction;

Technical Subject Area. Mobile Internet: Applications and Technology, Personal Communications

1. Introduction. With recent advances in wireless standards, such as the IMT2000 for cellular networks as well as the HIPERLAN and the IEEE802.11 standards for wireless local area networks, there has been great expectation that wireless data applications will soon become popular just like wireless telephony. In anticipation of this development, there have been many attempts in testing pilot applications on various wireless platforms. The Information Engineering Department at the Chinese University of Hong Kong has established a site, jawap.net, based on the Wireless Application Protocol(WAP) to provide a host of wireless applications, including an implementation of a Dutch Auction System.

The Dutch auction is said to originate in the Netherlands and uses a descendingprice format unlike the so-called "English Auction". In the Dutch scheme, when an object is presented to interested buyers for bidding the price will start at a high value and progressively decreases downward until a buyer bids for the object by making a declaration. If multiple declarations are made, any common resolution scheme can be invoked to break the tie. It is possible to extend this scheme for the auctioning of multiple units of an object. Successful bidders bidding at the same price will each receive their unit at the bid price. If the number of units is not sufficient to cover

\footnotetext{
${ }^{*}$ Received on May 22, 2002; accepted for publication on October 21, 2002. This work was partially supported by a grant from the Area of Excellence in Information Technology in the Hong Kong Special Administrative Region.

$\dagger$ WINLAB, Rutgers University, Piscataway, NJ 08854-8060, USA. E-mail: andyyuen@winlab.rutgers.edu

${ }^{\ddagger}$ Department of Computer Engineering and Information Technology, City University of Hong Kong, Tat Chee Avenue, Hong Kong. E-mail: itcwsung@cityu.edu.hk

${ }_{\S}^{\S}$ Department of Information Engineering, Chinese University of Hong Kong, Shatin, N.T., Hong Kong. E-mail: wswong@ie.cuhk.edu.hk
} 
all the bids, a tie-breaking rule is invoked. If sufficient units of the object are still available, the auction will continue until all the units are sold or a reserved price level is reached.

The Dutch auction is one of the four major auctioning schemes ([1] or the survey article [2],) that also include the English auction ${ }^{1}$, first price sealed bid auction ${ }^{2}$, and second price sealed bid auction (also known as the Vickrey auction) ${ }^{3}$. The seminal work of Vickrey [3] analyzed and compared these four common kinds of auction rules. Since the process of an auction is very complicated involving the auctioneer, the seller, and multiple bidders, it is common to make certain simplifying assumptions about these players in order to make the analytical model tractable. A key concept concerns the idea of the value of the object under auction. The value can be private, that is, a person buys an item for his/her own consumption without an objective to resell, or common, in which case the buyer bids with the intention of resell and has to estimate the valuation offered by prospective buyers. In the latter case, the competitors are clearly a helpful source to obtain such a valuation estimate. A bidder is said to be risk neutral if he/she bids exactly accordingly to his/her evaluation of the object. A bidder who is likely to bid above his/her evaluation to increase the chance of winning is called a risk averse bidder.

Vickrey's paper [3] assumes that each bidder is risk-neutral and knows the value of the object to himself/herself but not the value to other bidders. Moreover, the model is assumed to consist of symmetric bidders, that is, individual valuation of the object is i.i.d. Since then, there has been a steady output of follow-up work on optimal auction design. One important piece of work was due to Myerson [4]. He extended the work of Vickrey in two directions. Firstly, the case of asymmetric bidders is considered, in which individual valuations are independent but not necessarily identically distributed. Secondly, different viable ways of selling the object was considered rather than just a prespecified set of auction rules. Under this framework, the optimal auction design problem to maximize the expected revenue of the auction host is solved. There have been many more additional works on the design of optimal auction rules. The well known auction rules are compared under various relaxations of the stated assumptions [5], [6], [7].

With the advent of the World Wide Web, online auctions have become increasingly popular. Moreover, the arrival of $3 \mathrm{G}$ and high speed wireless local area networks have made the idea of hosting auctions to serve mobile users via wireless communica-

\footnotetext{
${ }^{1}$ In the English auction, bidders compete with each other by offering progressively higher bids until one bidder remains, who is committed to buy at last price of the last bid.

${ }^{2}$ In a sealed bid auction, all the buyers submit a bid at the same time. The buyer offering the highest bid is committed to buy the object the bid price.

${ }^{3}$ In such an auction, the buyer making the highest bid is committed to buy the object, but at a price equal to the second highest bid.
} 
tion devices, such as enhanced cellular phones or personal digital assistants, practical in the near future. Coupled with the concept of micropayment, one can envision the possibility of auctioning all sorts of items which may have only small monetary value or are time-critical, such as tickets for an upcoming concert or seats on air flights.

For these applications, the expected time for completing an auction and the amount of signaling messages needed to conduct an auction are important consideration factors. These two elements are not considered in classical auctioning models. For Internet based auctions, in particular those available on wireless accesses, the issue of communication cost cannot be ignored. From this perspective, the Dutch auctioning scheme intuitively has an advantage over the English auctioning scheme. For the former scheme, no bidder is required to bid more than once, whereas for the latter scheme bidders may have to bid several times whether they are successful at the bidding or not. Moreover, although the possibility of multiple bids at the same value occurs in both cases, it can happen at most once in the auctioning process for an item in a Dutch scheme and multiple times for the English scheme.

Another motivation of our work comes from the observation that in the literature, a common assumption is that the bidding offers take values from a continuum. This is an idealization of an actual bidding process. In practice, bid increments in an English auction or bid decrements in a Dutch auction is a discontinuous process. While a small discontinuous price decrement would minimize inaccuracies due to discrete optimization, it would also prolong the auctioning process. For auctions conducted over the Internet, the value of a discontinuous decrement could have significant implications on the communication cost. In particular, for a Dutch auction, the optimal strategy for price decrement is an interesting issue.

In this paper, we present an analysis of an Internet based Dutch auctioning system. In any auction, there are three player roles that one can consider, the buyer, the seller, and the auction host. Traditional analyses on auctioning tend to focus on the roles played by the buyer or the seller. The role of the auction host is defined in terms of the type of auctioning system used. For auctions conducted on Internet, the auction host is bestowed with a new set of controlling mechanisms and faces a new set of objectives. Assuming the revenue of the auction host comes from commission based on the realized bid revenue and the varying part of an auctioning cost is proportional to the duration of the bidding process, one can formulate an objective function based on these two factors. In a Dutch auction, an important controlling mechanism available to an auction host is through price decrement strategy. The structure of the optimal price decrement strategy in an Internet based Dutch auctioning system is analyzed here via the Karush-Kuhn-Tucker condition. Moreover, we also established a numerically efficient algorithm to determine the optimal strategy. Numerical studies were carried out and we showed that under certain conditions, the simple uniform decrement strategy can be close to the optimal strategy. These results form a small, 
first step to generalize the earlier works in the literature on auction in the new context of the Internet based environment.

The rest of the paper is organized as follows. In section 2, we describe the system and optimization model. We maximize the expected revenue to the auction host by dynamically varying price decrement at each iteration. Knowledge on the number of bidders and the probability distributions of their valuations are exploited. In section 3, properties of the optimal solution are presented. We also show how the original problem can be reduced to a one dimensional numerical search problem. In section 4, we present numerical examples to illustrate the properties of the optimal solution. Performance comparison between the optimal strategy and a simple uniform decrement strategy is also given. Section 5 offers some concluding remarks.

\section{System and Optimization Model.}

2.1. System Model. In an auction, there are three distinct player roles, namely the buyer, seller and auction host. In an Internet based auction, the auction host usually acts as the application server and provides the necessary information to implement the auction. It disseminates current price information to all bidders (logon users) regularly, and ends the auction when it receives a buying request from the users or the auction timeout is reached.

We assume only one item is sold in the auction. Initially, the auction starts at a given price $c_{0}$. A price is kept constant for a fixed interval until the next iteration. At iteration $k$, the price falls to $c_{k}$, under the constraint $c_{\min } \leq c_{k} \leq c_{k-1}$. The auction will last for $M+1$ iterations, where $M$ is predetermined.

Denote $X_{i}$ as the valuation of bidder $i$. Let $n$ be the number of bidders. Since the auction host is also the application server it knows the value of $n$. In this work, we assume $n$ is constant for the duration of the auction. We also assume the valuations of the $i^{t h}$ bidder, $X_{i}$, for all $i \in[1, n]$ are i.i.d. random variables drawn from a known distribution $F_{X}($.$) . In literature this is known as the case of symmetric bidders.$ However, our solution methodology also works for asymmetric bidders. This is the case where bidder valuations are drawn from independent but not necessarily identical distributions.

Also denote $Y=\max \left(X_{1}, \ldots, X_{n}\right)$ as the maximum valuations of the bidders. It is straightforward to compute the cumulative distribution function $c d f$ and probability density function $p d f$ of $Y$ for the cases of symmetric or asymmetric bidders. We denote them by the notation $F(Y)$ and $f(Y)$ respectively. For simplicity, we assume that $f(Y)$ is a continuous positive function in the range $c_{m i n}, c_{0}$. Subsequently we will work with $Y$ directly since the sold price depends on the random variable $Y$. If the current selling price $c_{k}$ is lower than $Y$, at least one user will immediately make a bid and end the auction. If $c_{M}>Y$, the item will not be sold at the auction. 
2.2. Optimization model. Suppose the item is sold at iteration $k . c_{k}$ is the selling price at iteration $k$, and $T$ is a non-negative time discounting increment at each iteration. Thus, the revenue is $R_{k}=c_{k}-k T$ if the item is sold at iteration $k, k \in\{0, \ldots, M\}$. If the item is not sold at the end of auction (i.e. $c_{M}>Y$ ), we define the corresponding revenue as $R_{M+1}=0$.

The expected revenue upon selling the item is

$$
\begin{aligned}
p(\mathbf{c}) & =\mathbf{E}_{k \in\{0, \ldots, M+1\}}\left[R_{k}\right] \\
& =\mathbf{E}_{k \in\{0, \ldots, M\}}\left[c_{k}-k T\right] \\
& =c_{0}\left(1-F\left(c_{0}\right)\right)+\sum_{k=1}^{M}\left(c_{k}-k T\right)\left(F\left(c_{k-1}\right)-F\left(c_{k}\right)\right) .
\end{aligned}
$$

In our optimization model, we incorporated a time discounting factor $T$. The meaning of $T$ can be interpreted in two different scenarios. First of all, $T$ can represent the cost of using server resources in a wireless Dutch auction. Typically, the auction period spans only for minutes or hours. The auction server may update the price on a per minute or second basis. At each iteration, the application server has to broadcast a message to update the current price to all the clients (bidders). This generates a lot of data traffic and uses up bandwidth resources. Moreover, the amount of processing and bandwidth overhead can be regarded as being constant at each iteration. Thus, the resource usage is adequately modeled by a constant parameter $T$.

Alternatively, this model can also apply to online auction websites where auction period spans over longer periods of time, such as days or weeks. It is common in these cases that the price of an item is dropped gradually on a daily basis. In this scenario, the amount of network traffic generated is insignificant. Rather, there is a time discounting factor on the value of the good to account for storage and maintenance cost incurred on the auction host.

The present problem belongs to a class of general nonlinear optimization problems with inequality constraints. The problem is to

$$
\max _{\left(c_{1}, c_{2}, \ldots, c_{M}\right)} p(\mathbf{c})=\max _{\left(c_{1}, c_{2}, \ldots, c_{M}\right)} c_{0}\left(1-F\left(c_{0}\right)\right)+\sum_{k=1}^{M}\left(c_{k}-k T\right)\left(F\left(c_{k-1}\right)-F\left(c_{k}\right)\right)
$$

subject to the constraints

$$
\begin{array}{ll}
g_{1}(\mathbf{c}) & =c_{1}-c_{0} \leq 0, \\
g_{2}(\mathbf{c}) & =c_{2}-c_{1} \leq 0, \\
\vdots & \\
g_{M}(\mathbf{c}) & =c_{M}-c_{M-1} \leq 0, \\
g_{M+1}(\mathbf{c}) & =c_{\text {min }}-c_{M} \leq 0 .
\end{array}
$$

We note that many alternative formulations such as dynamic programming [8] are possible. Our nonlinear programming formulation is desirable for practical imple- 
mentation because, as we will show in the next section, the multivariable optimization problem of determining the selling price $M$-tuple $c_{k}, k=1,2, \ldots, M$ can be reduced to a one dimensional numerical search problem. Since an auction host typically has hundreds or thousands of items for sale, the reduction of computation complexity in price setting is desirable for running a large auction hosting site.

3. Properties of the optimal solution. In the literature, optimization of nonlinear functions subject to inequality constraints is well studied. The KarushKuhn-Tucker (KKT) Theorem is one of the powerful tools commonly employed.

Let $\mathbf{c}$ be any point in the feasible set. Denote $J(\mathbf{c})=\left\{j: g_{j}(\mathbf{c})=0\right\}$. If $\nabla g_{j}(\mathbf{c})$ are mutually linearly independent for all $j \in J(\mathbf{c})$, then $\mathbf{c}$ is a regular point. The well known theorem due to Karush,Kuhn and Tucker [9] provides a necessary condition for a point to be a local maximizer, commonly known as the Karush-Kuhn-Tucker (KKT) condition, presented as follows.

Let $\mathbf{c}^{*}$ be a regular point and local maximizer for the problem of maximizing $p$ subject to $\mathbf{g}(\mathbf{c}) \leq 0$. Then there exists a vector $\mathbf{u}^{*} \in \Re^{M+1}$ such that

$$
\begin{aligned}
\mathbf{u}^{*} & \geq 0 \\
\nabla p\left(\mathbf{c}^{*}\right) & =\nabla \mathbf{g}\left(\mathbf{c}^{*}\right) \mathbf{u}^{*} \\
\mathbf{u}^{* T} \mathbf{g}\left(\mathbf{c}^{*}\right) & =0
\end{aligned}
$$

where

$$
\mathbf{u}^{*}=\left(\begin{array}{c}
u_{1}^{*} \\
u_{2}^{*} \\
\vdots \\
u_{M+1}^{*}
\end{array}\right) \quad \mathbf{g}\left(\mathbf{c}^{*}\right)=\left(\begin{array}{c}
g_{1}\left(\mathbf{c}^{*}\right) \\
g_{2}\left(\mathbf{c}^{*}\right) \\
\vdots \\
g_{M+1}\left(\mathbf{c}^{*}\right)
\end{array}\right)
$$

and $\nabla \mathbf{g}\left(\mathbf{c}^{*}\right)$ is the $M \times M+1$ matrix whose $i$-th column is $\nabla g_{i}\left(\mathbf{c}^{*}\right)$.

We refer to the vector $\mathbf{u}^{*}$ as the Karush-Kuhn-Tucker (KKT) multiplier vector. In the literature, a point satisfying the KKT condition (equation 5-7) is called a critical point. It follows from the KKT Theorem that a local maximizer is a critical point but not necessarily vice versa. We also define the global maximum in the feasible set as $\mathbf{c}^{* *}$. Thus, if $\mathbf{c}^{* *}$ is regular, it is also within the set of all critical points.

For the stated optimization problem, the constraint stated by equation 4 can be rewritten as:

$$
\mathbf{g}(\mathbf{c})=\left(\begin{array}{c}
c_{1}-c_{0} \\
c_{2}-c_{1} \\
\vdots \\
c_{\min }-c_{M}
\end{array}\right)
$$


Therefore,

$$
\nabla \mathbf{g}(\mathbf{c})=\left(\begin{array}{rrrrrrr}
1 & -1 & 0 & \cdots & 0 & 0 & 0 \\
0 & 1 & -1 & \cdots & 0 & 0 & 0 \\
0 & 0 & 1 & \cdots & 0 & 0 & 0 \\
\vdots & \vdots & \vdots & \ddots & \vdots & \vdots & \vdots \\
0 & 0 & 0 & \cdots & -1 & 0 & 0 \\
0 & 0 & 0 & \cdots & 1 & -1 & 0 \\
0 & 0 & 0 & \cdots & 0 & 1 & -1
\end{array}\right)
$$

It is obvious that any selection of $M$ column vectors from $\nabla \mathbf{g}$ are mutually linearly independent. Hence any point, $\mathbf{c}$, in the feasible set with the cardinality of $J(\mathbf{c})$ less than $M+1$ is a regular point. The case where $J(\mathbf{c})=\{1,2, \ldots, M+1\}$ corresponds to $c_{0}=c_{1}=\ldots=c_{M}=c_{\text {min }}$, does not belong to the feasible set since $c_{0} \neq c_{\text {min }}$. Thus, every point in the feasible set is regular. It follows from KKT Theorem that all local maxima are regular and should satisfy the KKT equations. As a result, $\mathbf{c}^{* *}$ can be found by searching over the set of all critical points. Hereafter, we denote a critical point by $\mathbf{c}^{*}$.

On substitution of $\mathbf{g}\left(\mathbf{c}^{*}\right)$ and $\nabla \mathbf{g}\left(\mathbf{c}^{*}\right)$ into equation 6 , one obtains

$$
\begin{aligned}
\nabla p\left(\mathbf{c}^{*}\right) & =\left(\begin{array}{llll}
\frac{\partial p}{\partial c_{1}}\left(\mathbf{c}^{*}\right), & \frac{\partial p}{\partial c_{2}}\left(\mathbf{c}^{*}\right), & \ldots, & \frac{\partial p}{\partial c_{M}}\left(\mathbf{c}^{*}\right)
\end{array}\right) \\
& =\left(\begin{array}{llll}
u_{1}^{*}-u_{2}^{*}, & u_{2}^{*}-u_{3}^{*}, & \ldots, & u_{M}^{*}-u_{M+1}^{*}
\end{array}\right) .
\end{aligned}
$$

The last KKT condition in equation 7 leads to the conclusion

$$
u_{k}^{*} g_{k}\left(\mathbf{c}^{*}\right)=u_{k}^{*}\left(c_{k}^{*}-c_{k-1}^{*}\right)=0, \quad \text { for } \quad k=1,2, \ldots, M+1 .
$$

since $c_{k}^{*}-c_{k-1}^{*}$ 's are non-positive for all $k$.

There are standard algorithmic approaches to solve the class of convex programming problems in which the objective function is concave and the feasible set is convex. However, we show in the appendix that our objective function is not concave in general. As a result, local search technique is applied to identify local maxima. The search procedure is repeated with different initial points to discover as many distinct local maxima as possible. The best of these local maxima is chosen as the solution. Numerical computations for this heuristic approach over the feasible set for local maxima can be quite extensive. This is an important consideration when the number of iterations is large. In this case an optimization problem in $M$ variables needs to be considered. However, it turns out that by exploiting our knowledge of the structure of the critical points, we could determine the global optimum $\mathbf{c}^{* *}$ by reducing the problem to a one dimensional search problem. This is the main result provided by theorem 3. In the following, we present some basic properties of the optimal solution and describe an iterative solution methodology for finding the global maximum $\mathbf{c}^{* *}$. 
Suppose one implements an auction following the optimal price vector $\mathbf{c}^{* *}$. At iteration $j$, the current price is $c_{j}^{* *}$. Define the subproblem starting at iteration $j$ as one in which there are $M-j$ remaining iterations, starting from the price $c_{j}^{* *}$. The problem of finding the optimal price vector for this problem is equivalent to solving the problem,

$$
\begin{gathered}
\max _{\left(c_{j+1}, \ldots, c_{M}\right)} \mathbf{E}_{k \in\{j+1, \ldots, M+1\}}\left[R_{k} \mid Y<c_{j}^{* *}\right] \\
=\max _{\left(c_{j+1}, \ldots, c_{M}\right)} \mathbf{E}_{k \in\{j+1, \ldots, M\}}\left[c_{k}-k T \mid Y<c_{j}^{* *}\right] .
\end{gathered}
$$

Proposition 1. For any $j, j \in\{1, \ldots, M-1\},\left(c_{j+1}^{* *}, c_{j+2}^{* *}, \ldots, c_{M}^{* *}\right)$ is the optimal price vector to subproblem starting at iteration $j$.

Proof.

$$
\begin{aligned}
& \max _{\left(c_{j+1}, c_{j+2}, \ldots, c_{M}\right)} \mathbf{E}_{k \in\{j+1, \ldots, M\}}\left[c_{k}-k T \mid Y<c_{j}^{* *}\right] \\
= & \max _{\left(c_{j+1}, c_{j+2}, \ldots, c_{M}\right)} \sum_{k=j+1}^{M}\left(c_{k}-k T\right) \frac{\left(F\left(c_{k-1}\right)-F\left(c_{k}\right)\right)}{F\left(c_{j}^{* *}\right)} \\
= & \frac{1}{F\left(c_{j}^{* *}\right)} \max _{\left(c_{j+1}, c_{j+2}, \ldots, c_{M}\right)} \sum_{k=j+1}^{M}\left(c_{k}-k T\right)\left(F\left(c_{k-1}\right)-F\left(c_{k}\right)\right) .
\end{aligned}
$$

It is obvious that the above expression is optimized when $c_{k}=c_{k}^{* *}$ for $k=$ $j+1, \ldots, M$.

The previous result states that if the number of bidders $n$ is constant throughout the auction period, then we need to compute $\mathbf{c}^{* *}$ only once at the start of the auction. In practice, $n$ may change from time to time as bidders may join or leave during an auction. In that case, one needs to get an update on the value of $n_{j}$ at iteration $j$ and computes the new optimal price vector.

When the number of iterations $M$ is large, numerical optimization becomes more complicated due to the number of variables involved. In theorem 1 and 3 , we show that the multivariable optimization problem can be reduced to a one dimensional search problem.

Theorem 1. Suppose $\mathbf{c}^{*}$ is a critical point and there exists an integer $i$ such that $c_{i}^{*}=c_{i+1}^{*}$, where $i=\{0,1, \ldots, M-1\}$. Then for all $j>i, j \in\{i+1, \ldots, M\}, c_{i}^{*}=c_{j}^{*}$.

Proof. Any feasible point must satisfy the condition:

$$
c_{0} \geq c_{1}^{*} \geq \ldots \geq c_{k}^{*} \geq c_{k+1}^{*} \geq \ldots \geq c_{M}^{*} \geq c_{\min }
$$

We claim that there does not exist an integer $i, 0 \leq i \leq M-2$, and a $j, i<j \leq M-1$, such that:

$$
c_{0}>c_{1}^{*}>\ldots>c_{i}^{*}=c_{i+1}^{*}=\ldots=c_{j}^{*}>c_{j+1}^{*} \geq \ldots \geq c_{M} \geq c_{\min } .
$$


We prove this statement by contradiction. By the Karush-Kuhn-Tucker Theorem we have to find $\mathbf{u}^{*} \geq \mathbf{0}$ so that equations 5-7 are satisfied. For convenience, we define the functions $h$ and $h_{M}$ as

$$
\begin{aligned}
& h\left(c_{k-1}, c_{k}, c_{k+1}\right)=\frac{\partial p}{\partial c_{k}} \\
& =F\left(c_{k-1}\right)-F\left(c_{k}\right)+f\left(c_{k}\right)\left(c_{k+1}-c_{k}-T\right) \quad k \in\{1, \ldots, M-1\}, \\
& h_{M}\left(c_{M-1}, c_{M}\right)=\frac{\partial p}{\partial c_{M}} \\
& =F\left(c_{M-1}\right)-F\left(c_{M}\right)+\left(c_{M}-M T\right)\left(-f\left(c_{M}\right)\right) .
\end{aligned}
$$

Since $g_{k}\left(\mathbf{c}^{*}\right)<0$ for $k \in\{1, \ldots, i\}$, by equation 12 ,

$$
u_{k}^{*}=0, \quad k \in\{1, \ldots, i\} .
$$

At the point $\mathbf{c}^{*}$, by equation 11 the following equations hold:

$$
\begin{aligned}
\frac{\partial p}{\partial c_{k}}\left(\mathbf{c}^{*}\right) & =h\left(c_{k-1}^{*}, c_{k}^{*}, c_{k+1}^{*}\right)=u_{k}^{*}-u_{k+1}^{*}=0, \quad k \in\{1, \ldots, i-1\} \\
\frac{\partial p}{\partial c_{i}}\left(\mathbf{c}^{*}\right) & =h\left(c_{i-1}^{*}, c_{i}^{*}, c_{i+1}^{*}\right)=-u_{i+1}^{*}, \\
\frac{\partial p}{\partial c_{i+1}}\left(\mathbf{c}^{*}\right) & =h\left(c_{i}^{*}, c_{i+1}^{*}, c_{i+2}^{*}\right)=u_{i+1}^{*}-u_{i+2}^{*}, \\
& \vdots \\
\frac{\partial p}{\partial c_{j-1}}\left(\mathbf{c}^{*}\right) & =h\left(c_{j-2}^{*}, c_{j-1}^{*}, c_{j}^{*}\right)=u_{j-1}^{*}-u_{j}^{*}, \\
\frac{\partial p}{\partial c_{j}}\left(\mathbf{c}^{*}\right) & =h\left(c_{j-1}^{*}, c_{j}^{*}, c_{j+1}^{*}\right)=u_{j}^{*}-u_{j+1}^{*} .
\end{aligned}
$$

Since

$$
g_{j+1}\left(\mathbf{c}^{*}\right)=c_{j+1}^{*}-c_{j}^{*}<0,
$$

equation 12 implies that $u_{j+1}^{*}=0$.

$$
\begin{aligned}
\frac{\partial p}{\partial c_{j}}\left(\mathbf{c}^{*}\right) & =u_{j}^{*}-u_{j+1}^{*}=u_{j}^{*} \\
& =h\left(c_{j-1}^{*}, c_{j}^{*}, c_{j+1}^{*}\right) \\
& =F\left(c_{j-1}^{*}\right)-F\left(c_{j}^{*}\right)+f\left(c_{j}^{*}\right)\left(c_{j+1}^{*}-c_{j}^{*}-T\right) \\
& =f\left(c_{j}^{*}\right)\left(c_{j+1}^{*}-c_{j}^{*}-T\right) \\
& <0 .
\end{aligned}
$$

That is $u_{j}^{*}<0$, hence the non-negativity condition on $\mathbf{u}^{*}$ is not satisfied. By contradiction we show that the scenario stated in equation 19 cannot hold.

COROllary 1. Suppose $\mathbf{c}^{*}$ is a critical point and

$$
c_{0}>c_{1}^{*}>\ldots>c_{i}^{*}=c_{i+1}^{*}=\ldots=c_{M}^{*} \geq c_{\min } .
$$


for some $0 \leq i<M$. Then

$$
\begin{array}{ll}
\frac{\partial p}{\partial c_{j}}\left(\mathbf{c}^{*}\right)=0 & j \in\{1, \ldots, i-1\}, \\
\frac{\partial p}{\partial c_{j}}\left(\mathbf{c}^{*}\right) \leq 0 & j \in\{i, \ldots, M-1\} .
\end{array}
$$

Proof. The proof of theorem 1 shows that

$$
\frac{\partial p}{\partial c_{i}}\left(\mathbf{c}^{*}\right)=-u_{i+1}^{*} \leq 0 .
$$

Since $c_{i}^{*}=c_{i+1}^{*}=\ldots=c_{M}^{*}$, we observe that

$$
\begin{aligned}
\frac{\partial p}{\partial c_{i+1}}\left(\mathbf{c}^{*}\right) & =\frac{\partial p}{\partial c_{i+2}}\left(\mathbf{c}^{*}\right)=\ldots=\frac{\partial p}{\partial c_{M-1}}\left(\mathbf{c}^{*}\right) \\
& =h\left(c_{i}^{*}, c_{i+1}^{*}, c_{i+2}^{*}\right) \\
& =F\left(c_{i}^{*}\right)-F\left(c_{i+1}^{*}\right)+f\left(c_{i+1}^{*}\right)\left(c_{i+2}^{*}-c_{i+1}^{*}-T\right) \\
& =-T f\left(c_{i+1}^{*}\right) \\
& \leq 0 .
\end{aligned}
$$

We now introduce the notation of a sequence-valued function

$$
\hat{\mathbf{c}}(s)=\left(\hat{c}_{0}, \hat{c}_{1}, \hat{c}_{2}, \ldots, \hat{c}_{M}\right)=\left(c_{0}, s, \hat{c}_{2}, \ldots, \hat{c}_{M}\right) .
$$

The domain for $s$ is defined in the range $c_{\min } \leq s \leq c_{0}$. The elements of $\hat{\mathbf{c}}$ are defined recursively in the following way:

Assume that elements up to $\hat{c}_{k}$ have been defined. Let $t$ be the solution to:

$$
h\left(\hat{c}_{k-1}, \hat{c}_{k}, t\right)=0 .
$$

(Note that by our assumption on the pdf and the definition of $h, t$ always exists and is unique.) If $c_{\min } \leq t \leq \hat{c}_{k}$ and $t-(k+1) T>0$, then $\hat{c}_{k+1}=t$. Otherwise, define $\hat{c}_{k+1}=\hat{c}_{k}$.

Note that $\hat{\mathbf{c}}(s)$ defines a 1-parameter family of critical points satisfying the KKT conditions. However, not all critical points can be represented by $\hat{\mathbf{c}}(s)$ for some $s$. Suppose $\mathbf{c}^{*}$ is a critical point and

$$
c_{0}>c_{1}^{*}>\ldots>c_{i}^{*}=c_{i+1}^{*}=\ldots=c_{M}^{*} \geq c_{\min }
$$

for some $0 \leq i<M$. If $c_{1}^{*}=\hat{c}_{1}$, then it follows directly from equation 25 and the definition of $\hat{\mathbf{c}}$ that

$$
c_{k}^{*}=\hat{c}_{k} \quad k \in\{1, \ldots, i-1\} .
$$

However, $c_{k}^{*}$ and $\hat{c}_{k}$, for $k \geq i$, may not be equal. In general,

$$
c_{k}^{*} \geq \hat{c}_{k} \quad k \in\{i, \ldots, M-1\} .
$$


To show that an optimal solution can be obtained by searching the family of critical points defined by $\mathbf{\mathbf { c }}(s)$, we need the following observation:

Theorem 2. Define $R_{j}=c_{j}^{* *}-j T$, for $j \in\{1, \ldots, M\}$. If $R_{j} \leq 0$, then $c_{k}^{* *}=c_{k-1}^{* *}$ for $k \in[j, M]$. If $R_{j}>0$, then $c_{j}^{* *}<c_{j-1}^{* *}$ if $c_{j-1}^{* *}>c_{\text {min }}$.

Proof. Suppose $R_{j}<0$. We have $R_{M} \leq R_{M-1} \leq \ldots \leq R_{j+1} \leq R_{j}<0$.

$$
\begin{aligned}
p(\mathbf{c}) & =\sum_{k=1}^{M} R_{k}\left(F\left(c_{k-1}\right)-F\left(c_{k}\right)\right) \\
& =\sum_{k=1}^{j-1} R_{k}\left(F\left(c_{k-1}\right)-F\left(c_{k}\right)\right)+\sum_{k=j}^{M} R_{k}\left(F\left(c_{k-1}\right)-F\left(c_{k}\right)\right) .
\end{aligned}
$$

If $c_{k}^{* *}<c_{k-1}^{* *}$ for any $k \in[j, M], p$ can be increased by setting $c_{k}^{* *}=c_{k-1}^{* *}$ for $k \in[j, M]$. A contradiction.

Suppose $R_{j}=0$ and $c_{j}^{* *}<c_{j-1}^{* *} . \quad p$ can be increased by changing $c_{j}^{* *}$ to any value in the interval $\left(c_{j}^{* *}, c_{j-1}^{* *}\right)$, and setting $c_{k}^{* *}=c_{k-1}^{* *}$ for $k \in[j+1, M]$. Again a contradiction.

On the other hand, suppose $R_{j}>0$. If $c_{j}^{* *}=c_{j-1}^{* *}$ then all $c_{k}^{* *}$ s must be equal for $k \geq j$ according to theorem 1 . Therefore, $p(\mathbf{c})$ can be increased by setting $c_{j}^{* *}$ to a value in the interval $\left(c_{\text {min }}, c_{j-1}^{* *}\right)$ while keeping $R_{j}>0$. A contradiction. Hence, $c_{j}^{* *}<c_{j-1}^{* *}$.

Now we are ready to prove our main result:

Theorem 3. If $\mathbf{c}^{* *}$ is an optimal solution, then $\hat{\mathbf{c}}(s)=\mathbf{c}^{* *}$ when $s=c_{1}^{* *}$.

Proof. Suppose $c^{* *}=\left(c_{0}^{* *}, c_{1}^{* *}, \ldots, c_{M}^{* *}\right)$ is an optimal solution. Set $s=c_{1}^{* *}$.

Define $R_{j}=c_{j}^{* *}-j T$. Suppose $R_{j}>0$ for all $j \in\{2, \ldots, M\}$, then it follows from corollary 1 and theorem 2 that $c_{j-1}^{* *}>c_{j}^{* *}$ and $h\left(c_{j-2}, c_{j-1}, c_{j}\right)=0$ unless $c_{j-1}^{* *}=c_{\text {min }}$. It follows from the definition of $\hat{\mathbf{c}}(s)$ that

$$
\hat{c}_{k}=c_{k}^{* *}
$$

for all $k$.

Suppose $R_{j} \leq 0$ for some $j \in\{2, \ldots, M\}$. If $j<M$, notice that $c_{M}^{* *}=c_{M-1}^{* *}=$ $\ldots=c_{j}^{* *}$ since $R_{k}<0$ for $k>j$. Hence, it follows from the definition of $\hat{\mathbf{c}}(s)$ that

$$
\hat{c}_{k}=c_{k}^{* *}
$$

for all $k$.

According to this theorem, by doing a one-dimensional numerical search for $\hat{\mathbf{c}}(s)$ within the feasible set, one can obtain an optimal solution to the problem. We now describe two more observations on the structure of the optimal solution. First of all, the following theorem shows that depending on whether $F($.$) is convex or concave, the$ sequence of price difference of the optimal strategy, $c_{k}^{* *}-c_{k+1}^{* *}$, satisfies the following inequalities. 
TheOREM 4. Given $\mathbf{c}^{* *}$ with the form $c_{0}>c_{1}^{* *}>\ldots>c_{i}^{* *}=c_{i+1}^{* *}=\ldots=c_{M}^{* *} \geq$ $c_{\text {min }}$. If $F($.$) is convex in \left[c_{\text {min }}, c_{0}\right]$, then

$$
c_{k-1}^{* *}-c_{k}^{* *} \leq c_{k}^{* *}-c_{k+1}^{* *}+T \quad k \in\{1, \ldots, i-1\} .
$$

If $F($.$) is concave in \left[c_{\min }, c_{0}\right]$, then

$$
c_{k-1}^{* *}-c_{k}^{* *} \geq c_{k}^{* *}-c_{k+1}^{* *}+T \quad k \in\{1, \ldots, i-1\} .
$$

Proof. By Corollary 1,

$$
\frac{\partial p}{\partial c_{k}}\left(\mathbf{c}^{* *}\right)=0 \quad k \in\{1, \ldots, i-1\} .
$$

That is,

$$
F\left(c_{k-1}^{* *}\right)-F\left(c_{k}^{* *}\right)+f\left(c_{k}^{* *}\right)\left(c_{k+1}^{* *}-c_{k}^{* *}-T\right)=0
$$

Consider the case when $F($.$) is convex.$

$$
\frac{F\left(c_{k-1}^{* *}\right)-F\left(c_{k}^{* *}\right)}{c_{k-1}^{* *}-c_{k}^{* *}} \geq f\left(c_{k}^{* *}\right)
$$

or

$$
F\left(c_{k-1}^{* *}\right)-F\left(c_{k}^{* *}\right)+f\left(c_{k}^{* *}\right)\left(c_{k}^{* *}-c_{k-1}^{* *}\right) \geq 0 .
$$

Subtracting equation 51 from equation 53 , we have

$$
f\left(c_{k}^{* *}\right)\left[\left(c_{k}^{* *}-c_{k-1}^{* *}\right)-\left(c_{k+1}^{* *}-c_{k}^{* *}-T\right)\right] \geq 0
$$

or

$$
c_{k-1}^{* *}-c_{k}^{* *} \leq c_{k}^{* *}-c_{k+1}^{* *}+T .
$$

The case when $F($.$) is concave can be proven in the same way.$

When $X$ is uniformly distributed as $U(a, b)$, the pdf of $Y$ is convex. In the special case $T=0$, we note that the price difference $c_{k}^{* *}-c_{k+1}^{* *}$ is increasing with time, whereas the probability $F\left(c_{k}^{* *}\right)-F\left(c_{k+1}^{* *}\right)$ is decreasing. This conforms to our intuition that price levels should be closely packed at intervals where pdf of $Y$ is large, such that the item could be sold at a price $c_{k}$ close to $Y$.

When $X$ is normal distributed as $N\left(\mu, \sigma^{2}\right)$,

$$
\begin{aligned}
& F(y)=Q\left(\frac{\mu-y}{\sigma}\right)^{n}, \\
& f(y)=n Q\left(\frac{\mu-y}{\sigma}\right)^{n-1} \frac{1}{\sqrt{2 \pi \sigma}} \exp \left(\frac{-(\mu-y)^{2}}{2 \sigma^{2}}\right) .
\end{aligned}
$$


It could be shown that $F(y)$ is convex when $y \leq \psi$ and concave otherwise, where $\psi$ is solution to the equation

$$
(n-1) \exp \left(\frac{-x^{2}}{2}\right)+\sqrt{2 \pi} x Q(x)=0, \quad x=\frac{(\mu-\psi)}{\sigma} .
$$

Since the pdf of $Y$ is largest at $\psi$, the price difference is decreasing at first and starts increasing again as $c_{k}^{* *}$ passes through $\psi$. That is, price levels are more closely packed around $Y=\psi$.

A uniform price decrement strategy is used as a reference in the numerical studies. The price vector starts at $c_{0}$ and falls to $c_{\min }$ in $M$ equally spaced steps. We hereafter refer this strategy as the uniform price decrement strategy. It turns out that this strategy is optimal in the trivial case as shown in the following theorem.

THEOREM 5. Uniform decrement strategy is optimal when

(1) $X \sim U(a, b), \quad c_{0} \leq b$ and $a \leq c_{\min }$

(2) $n=1$,

(3) $T=0$.

The optimal price levels are given by

$$
\begin{aligned}
c_{k}^{* *} & =\left(\frac{M-k}{M}\right) c_{0}-\left(\frac{k}{M}\right) c_{\text {min }}, & c_{\text {min }} & \geq \frac{c_{0}}{M+1}, \\
c_{k}^{* *} & =\left(\frac{M+1-k}{M+1}\right) c_{0}, & c_{\text {min }} & \leq \frac{c_{0}}{M+1} .
\end{aligned}
$$

Proof. We will show that $\mathbf{c}^{* *}$ defined in equation 59 and equation 60 satisfies the KKT conditions. Then we prove that $p$ is concave in the feasible set. Since $p$ is concave, the optimality of $\mathbf{c}^{* *}$ is proved.

$$
F(y)=\left\{\begin{array}{ll}
\frac{y-a}{b-a} & a \leq y \leq b \\
0 & \text { otherwise }
\end{array} \quad f(y)=\frac{1}{b-a} \quad a \leq y \leq b .\right.
$$

Substitute to equation 3 we have

$$
p=\frac{1}{b-a} \sum_{k=1}^{M} c_{k}\left(c_{k-1}-c_{k}\right)
$$

Taking partial derivatives w.r.t. $c_{k}$

$$
\begin{aligned}
\frac{\partial p}{\partial c_{k}} & =\frac{1}{b-a}\left[\left(c_{k-1}-c_{k}\right)-\left(c_{k}-c_{k+1}\right)\right] \quad k \in\{1, \ldots, M-1\}, \\
\frac{\partial p}{\partial c_{M}} & =\frac{1}{b-a}\left(c_{M-1}-2 c_{M}\right) .
\end{aligned}
$$

Consider the case $c_{\min } \geq \frac{c_{0}}{M+1}$. Substitute the price vector $\mathbf{c}^{* *}$ (equation 59) to 
equation 62 , we have

$$
\begin{aligned}
\frac{\partial p}{\partial c_{k}}\left(\mathbf{c}^{* *}\right) & =0 \quad k \in\{1, \ldots, M-1\} \\
\frac{\partial p}{\partial c_{M}}\left(\mathbf{c}^{* *}\right) & =\frac{1}{b-a}\left[-c_{\min }+\left(\frac{c_{0}-c_{\text {min }}}{M}\right)\right] \\
& =\frac{1}{b-a}\left[\frac{M-1}{M}\left(\frac{c_{0}}{M+1}-c_{\text {min }}\right)\right] \\
& \leq 0 .
\end{aligned}
$$

$g_{k}\left(\mathbf{c}^{* *}\right)<0 \quad \forall k \in\{1, \ldots, M\}$. By construction $u_{k}^{*}=0, k \in\{1, \ldots, M\}$, so that equation 12 is satisfied. Moreover, $u_{M+1}^{* *} \geq 0$. Thus we have

$$
\begin{aligned}
\frac{\partial p}{\partial c_{k}}\left(\mathbf{c}^{* *}\right) & =u_{k}^{* *}-u_{k+1}^{* *}=0 \quad k \in\{1, M-1\} \\
\frac{\partial p}{\partial c_{M}}\left(\mathbf{c}^{* *}\right) & =u_{M}^{* *}-u_{M+1}^{* *} \\
& =-u_{M+1}^{* *} \leq 0 .
\end{aligned}
$$

Thus the KKT condition 2 is also satisfied. Therefore there exists a non-negative $\mathbf{u}^{*}$ that satisfies all the KKT conditions.

Consider the case $c_{\min } \leq \frac{c_{0}}{M+1}$. We substitute the price vector $\mathbf{c}^{* *}$ (equation 60) to equation 62 , yielding

$$
\begin{aligned}
\frac{\partial p}{\partial c_{k}}\left(\mathbf{c}^{* *}\right) & =0 \quad k \in\{1, \ldots, M-1\} \\
\frac{\partial p}{\partial c_{M}}\left(\mathbf{c}^{* *}\right) & =\frac{1}{b-a}\left(c_{M-1}^{* *}-2 c_{M}^{* *}\right) \\
& =0
\end{aligned}
$$

on simplification. Since $g_{k}\left(\mathbf{c}^{* *}\right)<0$ for $k \in\{1, \ldots, M+1\}$, by equation $12 u_{k}^{* *}=0, k \in$ $\{1, \ldots, M+1\}$. Thus we have $u_{k}^{* *}-u_{k+1}^{* *}=0$. All the KKT conditions are satisfied again for this $\mathbf{c}^{* *}$.

To show that $\mathbf{c}^{* *}$ is a global maximizer, we proceed to prove the Hessian matrix $\mathbf{H}$ for the objective function $p$ is negative semi-definite. It is trivial to show that

$$
\mathbf{H}=\frac{1}{b-a}\left(\begin{array}{rrrrrr}
-2 & 1 & 0 & \ldots & \ldots & 0 \\
1 & -2 & 1 & 0 & \ldots & 0 \\
0 & 1 & -2 & 1 & \ldots & 0 \\
& & \ddots & \ddots & \ddots & 0 \\
0 & \ldots & 1 & -2 & 1 & 0 \\
0 & \ldots & 0 & 1 & -2 & 1 \\
0 & \ldots & \ldots & 0 & 1 & -2
\end{array}\right) .
$$

Apply the Gerschgorin's theorem on each row of $\mathbf{H}$, we show that $\max (\lambda) \leq 0$. Thus, $\mathbf{H}$ is negative semi-definite and $p$ is concave. 
So far Theorem 3 is the most important observation. Suppose an auction host knows the statistics of the individual valuation $F$. The auction host only needs to search for different values of $s$ for the optimal value $c_{1}^{* *}$. The nature of the optimization problem stipulates that if $s=c_{1}^{* *}$, then $\mathbf{c}^{* *}=\hat{\mathbf{c}}(s)$ is the optimum solution to the optimization problem. Given $s, \hat{\mathbf{c}}(s)$ can be determined easily by recursively solving simple algebraic equations (41) $M-1$ times. Thus, an exhaustive search of $s$ leads to the solution for the optimal price settings.

4. Numerical Studies. In this section, we firstly present several numerical examples to illustrate the properties of $\mathbf{c}^{* *}$. Then, the optimal strategy is compared to the uniform price decrement strategy in the following subsection.

4.1. Illustration of properties of optimal solution. In figure $1, X$ is uniformly distributed as $U(700,1000)$. There is no discounting factor, i.e. $T=0$. The optimal price $c_{k}^{* *}$ at iteration $k$ is plotted for $n=1,5,10,20,50$ respectively. When $n=1$, we observe that the uniform decrement strategy is optimal. Since $c_{\min } \geq \frac{c_{0}}{M+1}$, $\mathbf{c}^{* *}$ is given by equation 59. As $n$ increases, the pdf of $Y$ shifts to the right. Thus the price decrement rate is more gradual. When $n=20, c_{M}$ is approximately equal to 870 . When $n=50, c_{M}$ is approximately equal to 930 . In both cases, we note that the probability $\operatorname{Pr}\left[Y \leq c_{M}\right]$ is very small. We also observe that the cost difference $c_{k}^{* *}-c_{k-1}^{* *}$ is increasing with $k$. The observation is in agreement to equation 49 since $F_{Y}(y)$ is convex.

In the following examples, we assume the valuation of a bidder $X$ is normal distributed with mean 850 and variance $50^{2}$. The pdf of maximum valuation $Y$ for different $n$ is shown on figure 2. Note that the pdf becomes more peaked and shifts to the right as $n$ increases. Figure 3 shows the case when $T=0$ and $X$ is normal distributed as $N\left(850,50^{2}\right)$. For all values of $n$, the price difference is decreasing at first and increasing towards the end. This agrees with our results for normal distributed $X$ 's, since $F(Y)$ changes from convex to concave as Y increases. When $n=1$, the pdf is maximum around $\mu=850$. Thus, the initial drop in price is fast. After that, the drop in price is about the same in each iteration. On the other hand, when $n=50$, the pdf is maximum around 960 and tends to zero around 900. Thus, the drop in price is slowest around 960 and becomes faster after it passes through the pdf maxima. Note that for $n=10,20,50$, the iterations end essentially at the point when the pdf is essentially zero.

In figure 4 , a discounting factor of $T=20$ is introduced in each iteration. The price $c_{k}^{* *}$ at iteration $k$ is plotted for the cases $n=1,5,10,20,50$. We observe the inclusion of a non-zero discounting factor $T$ leads to faster price decrements. As one can read from figure 2, the maxima of $f(Y)$ occur around 850, 900,920,940,960 respectively when $n=1,5,10,20,50$. The optimal price decrement $c_{1}^{* *}$ lies in the vicinity of these pdf maxima. We note that when $n=50$, the simulation result is 


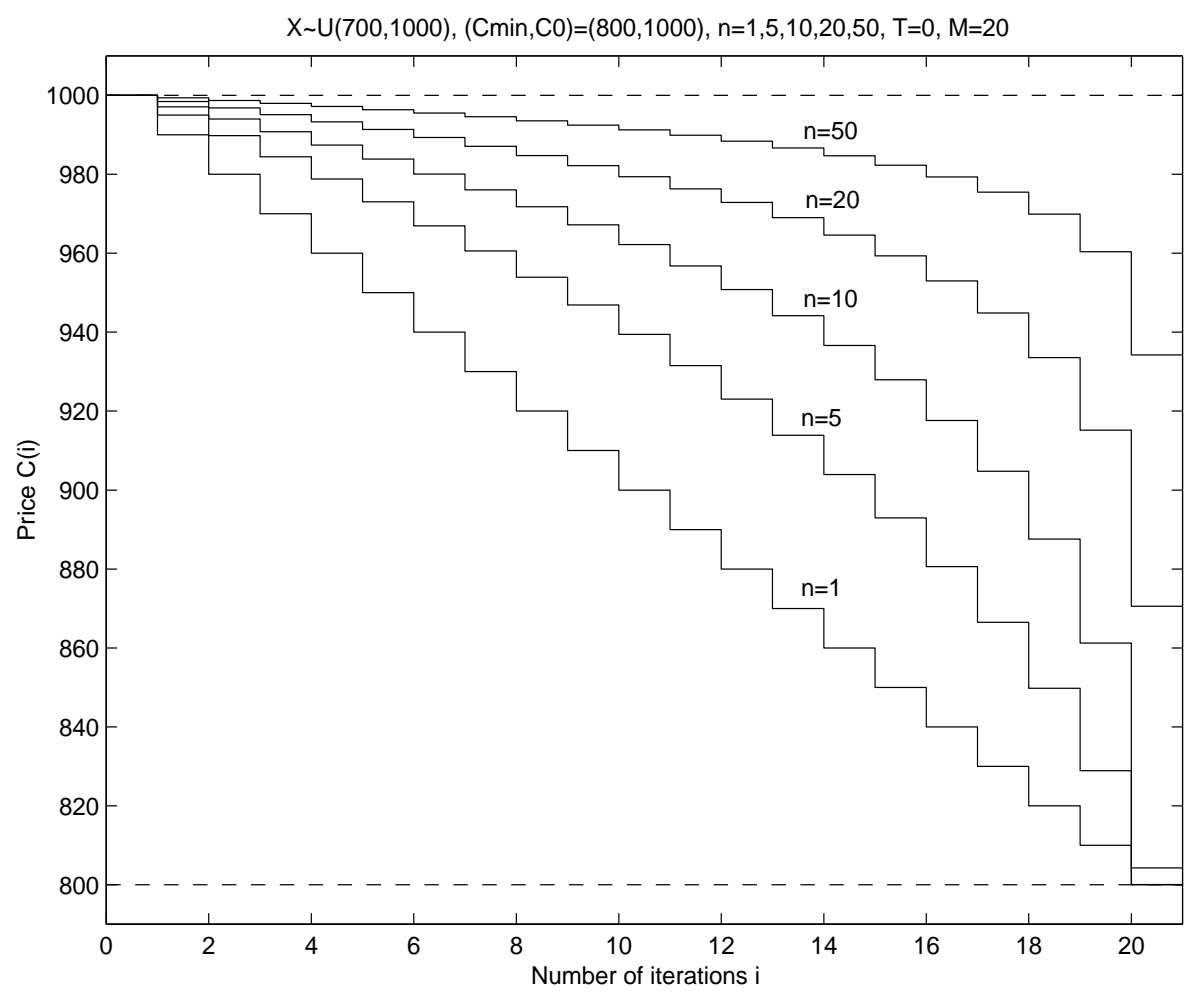

FIG. 1. Example 1: $X$ uniformly distributed, $T=0$

suboptimal. The auction ends at iteration 15 at a price higher than $c_{\min }$. Thus, the expected revenue can be further increased by additional price decrements. The discrepancy is due to numerical inaccuracies that occur at iterations when the pdf of $Y$ at the selling price is extremely small. In this case, the pdf of $\mathrm{Y}$ around $c_{15}$ is less than $10^{-9}$. Despite the numerical inaccuracies, the proposed method tends to yield solutions that are nearly optimal since the difference in the expected revenue is small.

In figure 5 , we change the discount factor to $T=50$. The optimal price decrements for different $n$ are shown. As predicted, the price decrement is even steeper compared to the cases where $T=20$ and $T=0$. In the case $n=20$ and $n=50$, the iterative solution fails to touch $c_{\min }$ when the auction ends due to the resolution inaccuracy in the search. Again, a nearly optimal solution is obtained since the pdf when the auction ends is very small (less than $10^{-10}$ ).

In figure 6 , the price decrements are compared for different discounting factor $T=0,5,10,20,50$. The number of bidders is $n=10$. As $T$ increases, the price decrement is steeper. Thus, if the resource usage is expensive, the auction host would prefer a strategy with faster price decrements. 


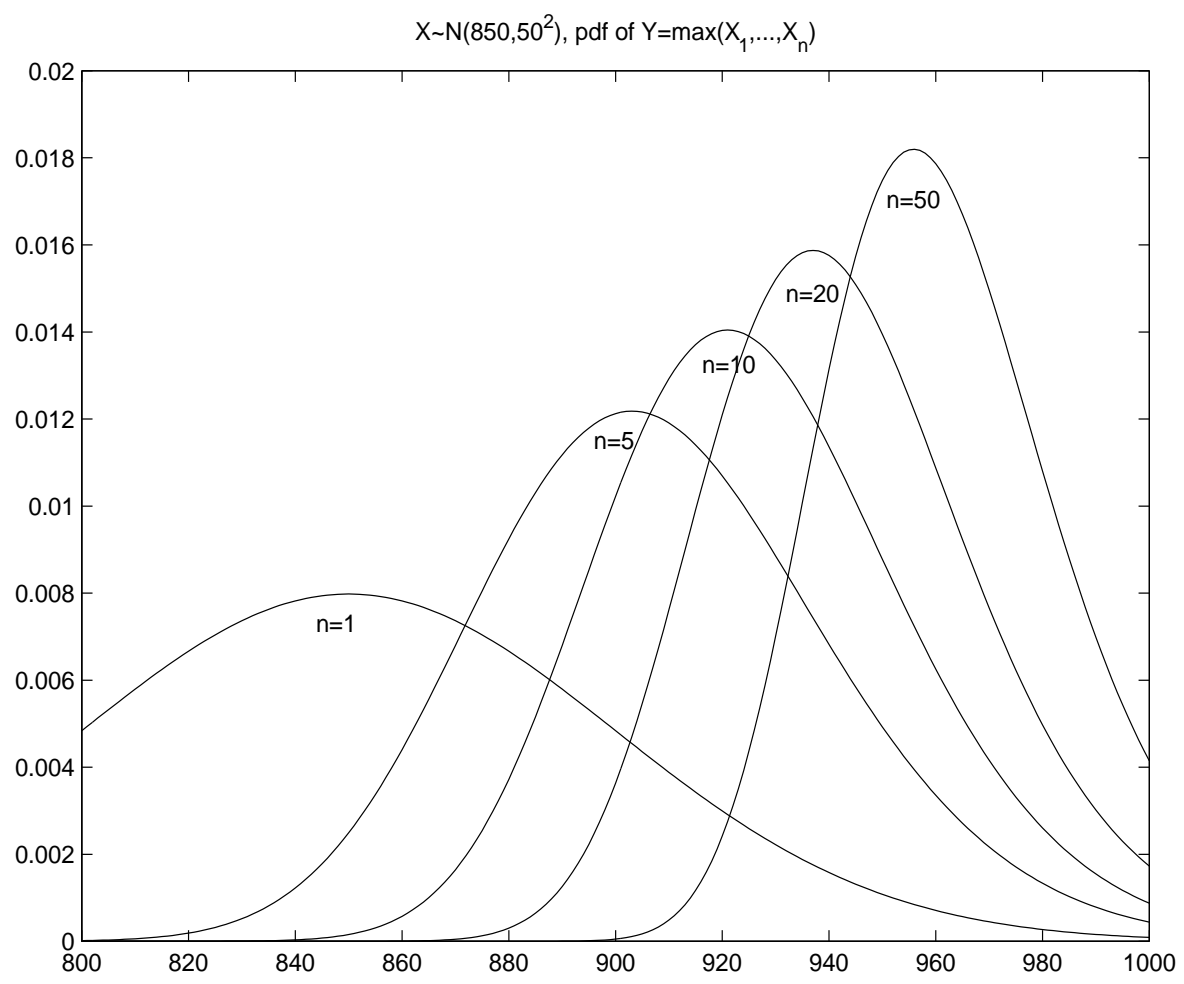

FIG. 2. pdf of $Y$ for $n=1,5,10,20,50$

TABLE 1

Revenue ratio of the optimal and the reference strategy when $T$ and $n$ are varied.

\begin{tabular}{|l|r|r|r|r|r|}
\hline$n$ & 1 & 5 & 10 & 20 & 50 \\
\hline \hline$T=0, X \sim U(700,1000)$ & 1.0000 & 1.0012 & 1.0027 & 1.0042 & 1.0058 \\
\hline$T=0, X \sim N\left(850,50^{2}\right)$ & 1.0009 & 1.0012 & 1.0018 & 1.0023 & 1.0028 \\
\hline$T=20, X \sim N\left(850,50^{2}\right)$ & 1.3920 & 1.2033 & 1.1444 & 1.1000 & 1.0566 \\
\hline$T=50, X \sim N\left(850,50^{2}\right)$ & 4.8749 & 1.9413 & 1.5764 & 1.3655 & 1.1948 \\
\hline
\end{tabular}

4.2. Comparison with the uniform decrement strategy. Comparison is done in terms of the expected revenue $p$. The ratio $p\left(\mathbf{c}^{* *}\right) / p\left(\mathbf{c}_{\text {ref }}\right)$ is shown in Table 1. We also compare the expected time to sell an item, as shown in Table 2. Suppose a strategy $\mathbf{c}$ is used. Define the expected time to sell an item $T_{s}$, given that it is actually sold when the auction ends as $\mathbf{E}\left[T_{s} \mid Y \geq c_{M}\right]$. It is straightforward to show that

$$
\begin{aligned}
\mathbf{E}\left[T_{s} \mid Y \geq c_{M}\right] & =\frac{\sum_{k=1}^{M} k\left(F\left(c_{k-1}\right)-F\left(c_{k}\right)\right)}{1-F\left(c_{M}\right)} \\
& =\frac{\sum_{k=0}^{M-1} F\left(c_{k}\right)-M F\left(c_{M}\right)}{1-F\left(c_{M}\right)} .
\end{aligned}
$$




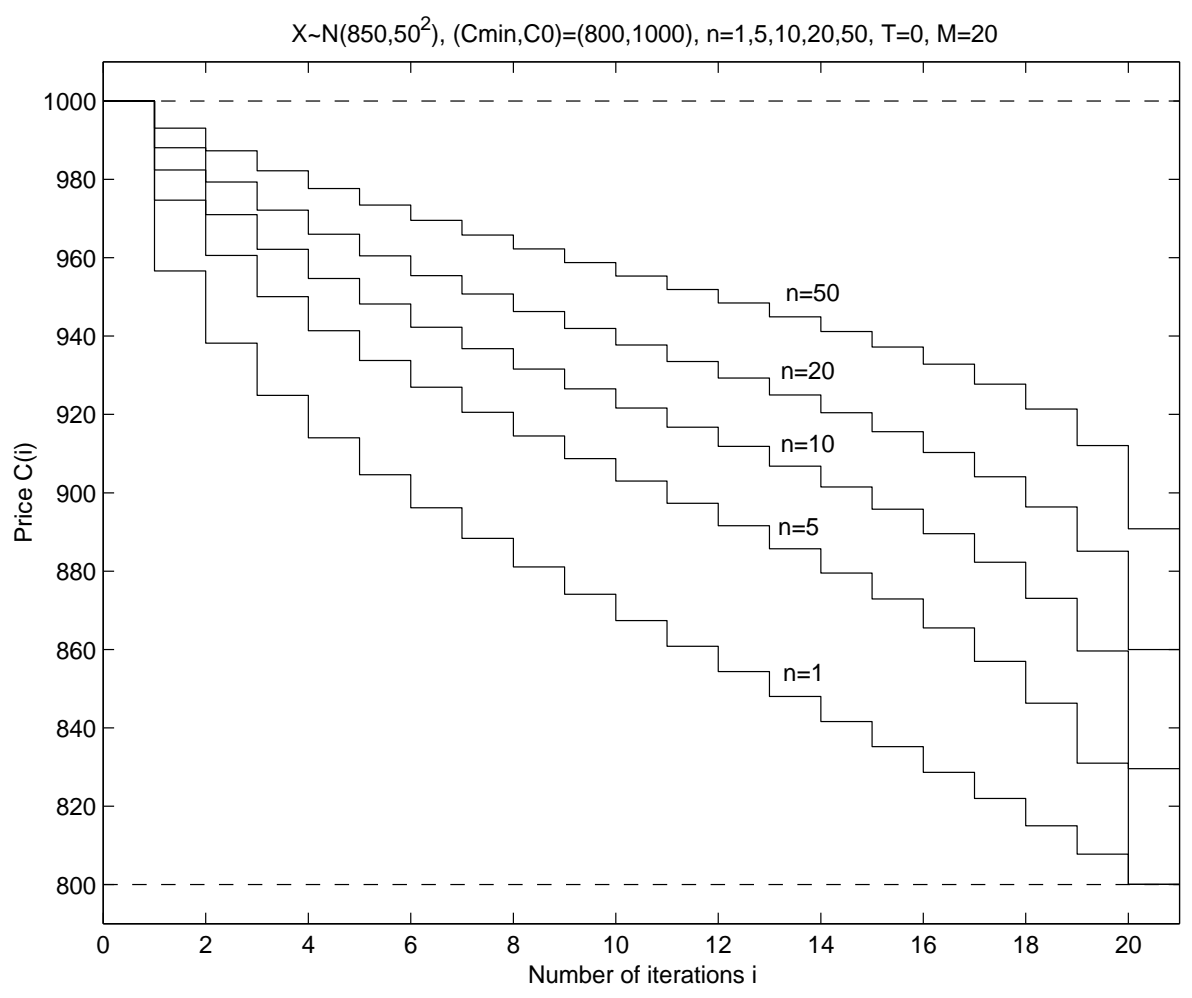

FIG. 3. Example 2: $X$ normal distributed, $T=0$

TABLE 2

Expected time to sell an item for the optimal and the reference strategy.

\begin{tabular}{|l|r|r|r|r|r|}
\hline$n$ & 1 & 5 & 10 & 20 & 50 \\
\hline \hline$T=0, X \sim U(700,1000)$ & $10.50 / 3.67$ & $8.10 / 5.42$ & $7.30 / 3.25$ & $7.06 / 1.98$ & $6.89 / 1.22$ \\
\hline$T=0, X \sim N\left(850,50^{2}\right)$ & $11.41 / 11.23$ & $9.87 / 9.69$ & $9.76 / 7.82$ & $9.57 / 6.19$ & $8.98 / 4.31$ \\
\hline$T=20, X \sim N\left(850,50^{2}\right)$ & $1.65 / 11.23$ & $1.57 / 9.69$ & $1.49 / 7.82$ & $1 / 41 / 6.19$ & $1.29 / 4.32$ \\
\hline$T=50, X \sim N\left(850,50^{2}\right)$ & $1.25 / 11.23$ & $1.24 / 9.69$ & $1.20 / 7.82$ & $1.15 / 6.19$ & $1.08 / 4.32$ \\
\hline
\end{tabular}

As illustrated in Table 1, the ratio of expected revenue for the optimal strategy over the reference strategy is shown. When $T=0$, the optimal strategy offers marginal improvement over the uniform price decrement strategy. This is true in general irrespective of the distribution of $X$. In general, the successful bidder will pay a little less than his valuation of the good under all price decrement strategies. The strategies are different mainly in the expected time that the product is sold. Since there is no discounting of revenue with time, the expected revenue of all strategies should appear the same. Applying this result to the context of wireless Dutch auction, we infer that a uniform decrement strategy is nearly optimal if the resource and processing overhead is low. 


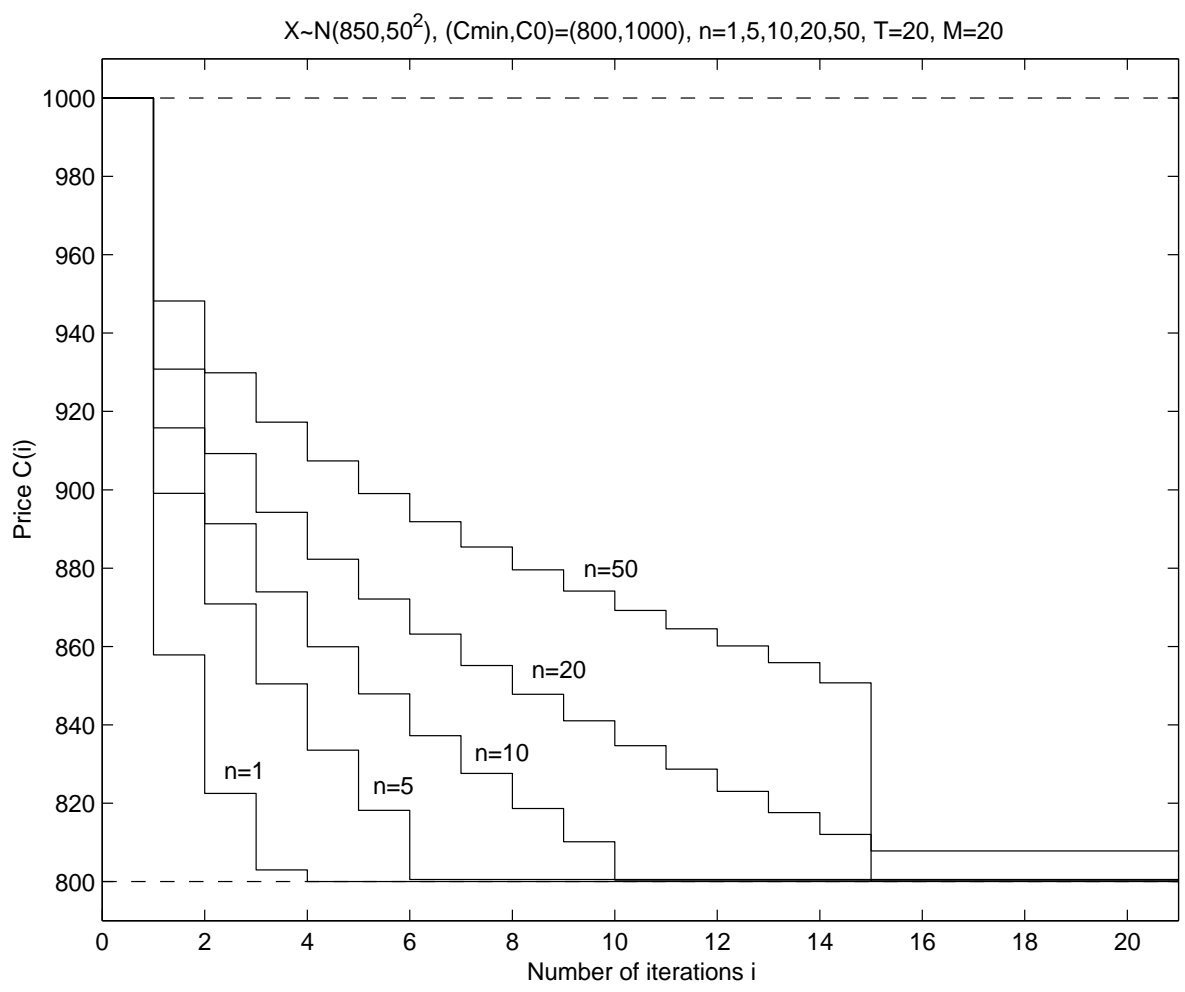

FIG. 4. Example 3: $X$ normal distributed, $T=20$

When the discounting factor is non-zero, the optimal strategy outperforms the alternate strategy by a large margin. In our studies, the difference is more remarkable when $n$ is small or $T$ is large. When $T$ is large, it is desirable to sell an item sooner to reap more profits. Reading from Table 2, the expected time to sell the good is much shorter for the optimal strategy. Thus, the optimal strategy is significantly better when $T$ is large. In a wireless Dutch auction, it is reasonable to assume the number of bidders $n=5$ or $n=10$. For the case $T=50$, the optimal strategy is superior to the uniform strategy by $94 \%$ and $57 \%$ respectively as read from Table 1 . Thus, in a wireless Dutch auction, we should refrain from using the uniform strategy if communication resources are expensive. Similarly, when the value of a good suffers from fast time discounting, as in perishable goods such as Dutch tulips, the uniform strategy should not be used.

From figure 2 we observe that the pdf shifts to the low price region when $n$ is small. For the uniform decrement strategy, it takes many iterations until the item is sold. Thus the revenue suffers from large time discounting. In the optimal strategy, the initial price decrements are steep so that the maximum valuation is reached upon several iterations. In practice, a Dutch auction is usually started at a very high initial price $c_{0} \gg Y>c_{m i n}$. The maximum valuation $Y$ is substantially below $c_{0}$. The use 


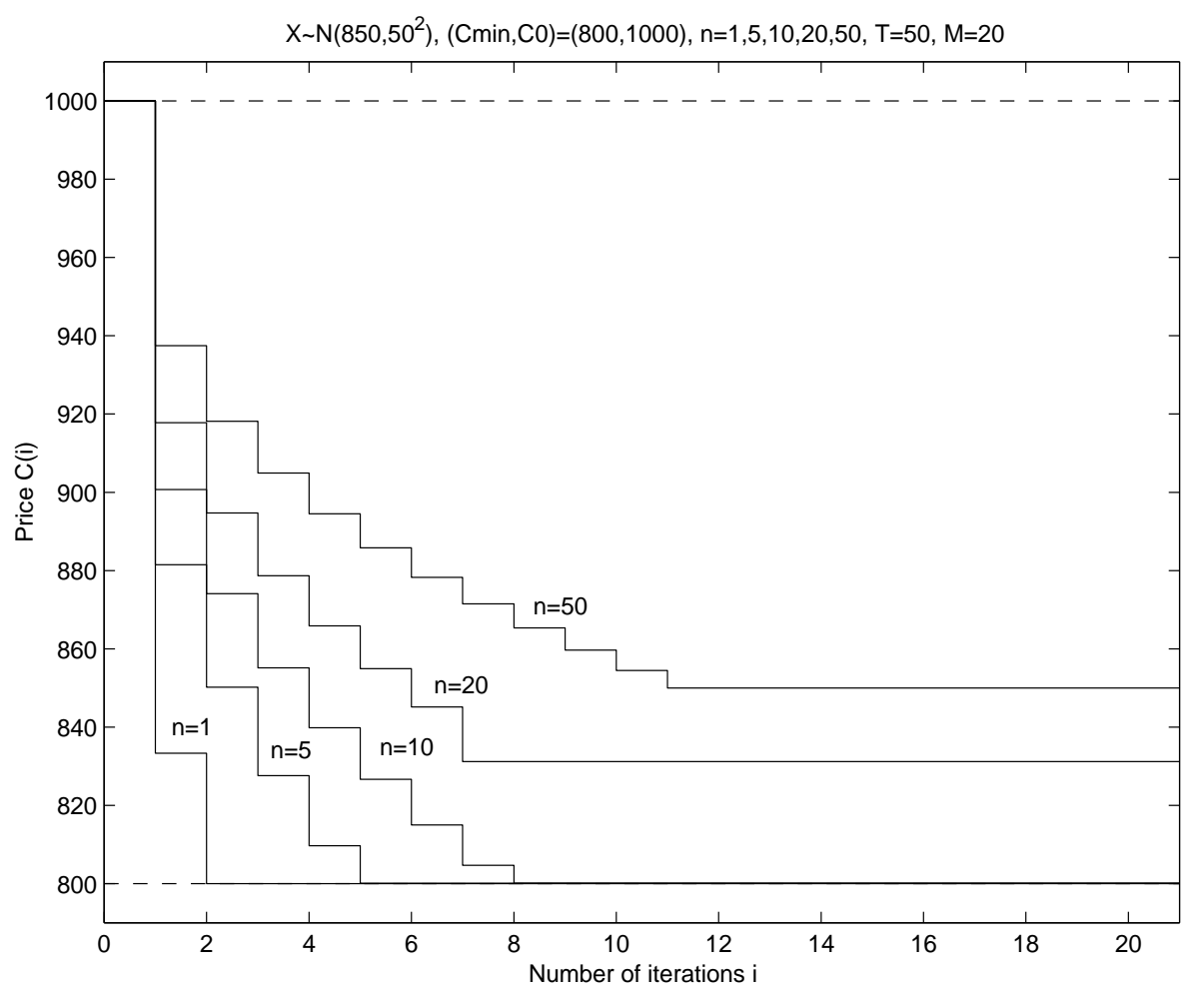

FIG. 5. Example 4: $X$ normal distributed, $T=50$

of the optimal strategy leads to significant gain over the uniform decrement strategy by significantly shortening the auction time and the corresponding amount of time discounting.

We have demonstrated that when the time discounting factor $T$ is large and when the number of bidders $n$ is small, the optimal strategy have the potential to outperform the uniform decrement strategy by a large margin. The underlying reason for the performance difference is that the auction time is significantly shortened for the optimal strategy as illustrated in Table 2. More generally, when parameters such as $c_{\text {min }}, \sigma$ and $M$ are varied, the auction time may be adversely prolonged by using the uniform decrement strategy. Thus under certain parameter settings, we also observe a large performance margin between the optimal and the uniform decrement strategy. Specifically, when the lower price limit $c_{\min }$ and individual valuations $X_{i}$ are small compared with the initial price $c_{0}$, the auction time for the reference strategy is considerably longer. Similarly, when the variance of the individual valuations $\sigma$ is small, the maximum valuation over all bidders $Y$ is also smaller, thus prolonging the auction time of the uniform decrement strategy. Finally, when the number of allowed iterations $M$ in an auction increases, the resultant price decrement interval of the uniform strategy is finer. This also adversely affect the performance of the reference 


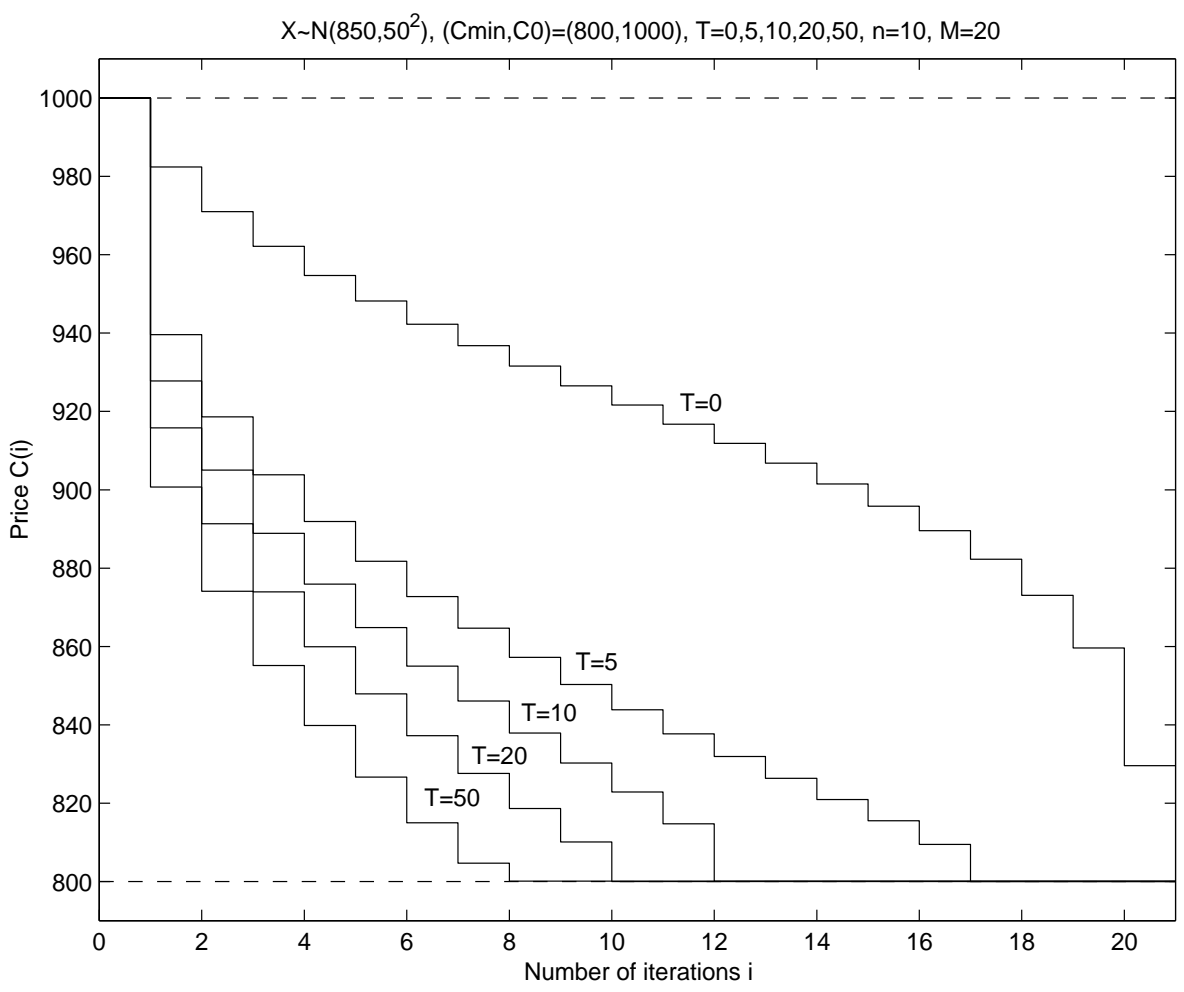

FiG. 6. Example 5: $X$ normal distributed, $n=10$

strategy relative to the optimal strategy.

TABLE 3

Revenue ratio of the optimal and the reference strategy when $c_{m i n}, \sigma$ and $M$ are varied.

\begin{tabular}{|l|r|}
\hline Parameters & revenue ratio \\
\hline \hline$T=10, n=10, c_{\text {min }}=100, M=20, \sigma=50, X \sim N\left(300,50^{2}\right)$ & 1.6168 \\
\hline$T=10, n=10, c_{\text {min }}=100, M=20, \sigma=100, X \sim N\left(300,50^{2}\right)$ & 1.3256 \\
\hline$T=10, n=10, c_{\text {min }}=100, M=20, \sigma=25, X \sim N\left(300,50^{2}\right)$ & 1.8736 \\
\hline$T=10, n=10, c_{\text {min }}=100, M=10, \sigma=25, X \sim N\left(300,50^{2}\right)$ & 1.5414 \\
\hline$T=10, n=10, c_{\text {min }}=100, M=30, \sigma=25, X \sim N\left(300,50^{2}\right)$ & 2.3685 \\
\hline
\end{tabular}

As a simple illustration we consider 5 more numerical examples with results shown in Table 3. In all the five examples, the lower price limit is $c_{\min }=100$. Individual valuations are modeled as i.i.d. Gaussian random variables with mean $\mu=300$ and variance $\sigma^{2}=50^{2}$. The revenue ratio of the optimal strategy relative to the uniform decrement strategy is found to be more than $60 \%$. This confirms our intuition that when $c_{0} \gg Y>c_{\text {min }}$, the optimal strategy outperforms the reference strategy by a large margin. In the second and third examples, the variance of the individ- 
ual valuations is varied as $\sigma^{2}=100^{2}$ and $\sigma^{2}=25^{2}$ respectively. The corresponding revenue ratios are 1.3256 and 1.8736 . Our results show that as the variance $\sigma^{2}$ increases, the revenue ratio also increases. When the valuations of the bidders show smaller randomness, it is unlikely that the maximum valuation is much higher than $\mu$. This decreases the efficiency of the uniform decrement strategy considerably. Finally, in the fourth and fifth examples we vary the number of iterations to $M=10$ and $M=30$ respectively. The corresponding revenue ratios are 1.5414 and 3.1962. This shows that when the number of iterations is large, the optimal strategy may lead to an improvement that is quite significant, as much as three times the revenue of the reference strategy.

5. Conclusion. In this paper, we present the optimal price decrement strategy for Dutch auction. It is shown in a system with inexpensive resources/low time discounting factor, the uniform decrement strategy is nearly optimal irrespective of the distribution of $X$. When resources are expensive/time discounting is high, the optimal strategy has steeper price decrements and is more favorable to the uniform strategy. Finally when the initial price is very high compared with the maximum valuation and the lower price limit, as in a practical Dutch auction, the optimal strategy is significantly better than the uniform strategy. We conclude that the optimal price decrement strategy is useful in a variety of contexts such as the wireless Dutch auction or online auction houses.

Appendix. In this appendix an argument to show why the objective function $p$ is not concave in general is described.

In order to render $p(\mathbf{c})$ concave, the Hessian matrix $\mathbf{H}=\left\{h_{k, j}\right\}$ must be negative semi-definite, where

Recall that

$$
h_{k, j}=\frac{\partial^{2} p}{\partial c_{j} \partial c_{k}} .
$$

$$
p(\mathbf{c})=\sum_{k=1}^{M}\left(c_{k}-k T\right)\left(F\left(c_{k-1}\right)-F\left(c_{k}\right)\right)+c_{0}\left(1-F\left(c_{0}\right)\right) .
$$

Taking partial derivatives with respect to $c_{k}$,

$$
\begin{aligned}
\frac{\partial p}{\partial c_{k}} & =F\left(c_{k-1}\right)-F\left(c_{k}\right)+f\left(c_{k}\right)\left(c_{k+1}-c_{k}-T\right) \quad k \in\{1, \ldots, M-1\} \\
\frac{\partial p}{\partial c_{M}} & =F\left(c_{M-1}\right)-F\left(c_{M}\right)+\left(c_{M}-M T\right)\left(-f\left(c_{M}\right)\right) .
\end{aligned}
$$

Differentiating w.r.t. $c_{j}$ again. For $k \in\{1, \ldots, M-1\}$, we have

$$
h_{k, j}=\left\{\begin{array}{rl}
f\left(c_{k-1}\right) & j=k-1 \\
-2 f\left(c_{k}\right)+f^{\prime}\left(c_{k}\right)\left(c_{k+1}-c_{k}-T\right) & j=k \\
f\left(c_{k}\right) & j=k+1 \\
0 & o . w .
\end{array}\right.
$$


Whereas, for $k=M$, we have

$$
h_{M, j}=\left\{\begin{aligned}
f\left(c_{M-1}\right) & j=M-1 \\
-2 f\left(c_{M}\right)+\left(-f^{\prime}\left(c_{M}\right)\right)\left(c_{M}-M T\right) & j=k \\
0 & \text { o.w. }
\end{aligned}\right.
$$

We observe that $\mathbf{H}$ is tri-diagonal with negative entries along the diagonals and positive entries adjacent to the diagonal entries. A sufficient condition to guarantee that $\mathbf{H}$ is negative semi-definite is to ensure its row sums are smaller than or equal to zero. However, this does not hold in general unless $T$ is very large.

To give an example, we consider the case where $M=1, n=1$, and $X$ is an exponential random variable with mean equal to 1 . In this case,

$$
p=c_{0}\left(1-F\left(c_{0}\right)+\left(c_{1}-T\right)\left(F\left(c_{0}\right)-F\left(c_{1}\right)\right) .\right.
$$

Differentiating this function twice, we have

$$
\frac{d^{2} p}{d c_{1}^{2}}=\left(c_{1}-T-2\right) e^{-c_{1}} \geq\left(c_{m i n}-T-2\right) e^{-c_{1}} .
$$

If $T<c_{\min }-2$, then $p$ is not concave.

To estimate the range of $T$ such that $p$ is concave, or $\mathbf{H}$ is negative semi-definite, one can use inclusion theorems on eigenvalues such as the Gerschgorin's theorem[11]. Since $\mathbf{H}$ is symmetric, all the eigenvalues are real. Applying the Gerschgorin's theorem on $\mathbf{H}$, each eigenvalue $\lambda_{i}$ must satisfy

$$
\lambda_{i} \leq h_{i, i}+\sum_{j=1, j \neq i}^{M}\left|h_{i, j}\right|
$$

in the feasible set. Thus $\mathbf{H}$ is negative semi-definite if $\max _{i} h_{i, i}+\sum_{j=1, j \neq i}^{M}\left|h_{i, j}\right| \leq 0$. On substitution, we have

$$
\begin{array}{r}
-f\left(c_{1}\right)+f^{\prime}\left(c_{1}\right)\left(c_{2}-c_{1}-T\right) \leq 0 \\
f\left(c_{k-1}\right)-f\left(c_{k}\right)+f^{\prime}\left(c_{k}\right)\left(c_{k+1}-c_{k}-T\right) \leq 0 \\
f\left(c_{M-1}\right)-2 f\left(c_{M}\right)-f^{\prime}\left(c_{M}\right)\left(c_{M}-M T\right) \leq 0 .
\end{array}
$$

Note that equation 78 holds in the feasible set. If equation 79 is true, then

$$
T \geq \frac{f\left(c_{k-1}\right)-f\left(c_{k}\right)}{f^{\prime}\left(c_{k}\right)}+\left(c_{k+1}-c_{k}\right)
$$

One can set $c_{k}=c_{k-1}=c_{0}$ and $c_{k+1}=c_{m i n}$ for example. Then $T \geq c_{0}-c_{\text {min }}$ must be satisfied to ensure $p$ is concave. 


\section{REFERENCES}

[1] www.agorics.com/new.html

[2] P. Klemperer, Auction Theory: A Guide to the Literature, Journal of Economical Surveys, 13:3(1999), pp. 227-284.

[3] W. Vickrey, Counterspeculation, Auctions and Competitive Sealed Tenders, Journal of Finance, 16(1961), pp. 8-37.

[4] R. B. Myerson, Optimal Auction Design, Mathematics of Operations Research, 6:1(1981), pp. $58-73$.

[5] Paul R. Milgrom and Robert J. Weber, A Theory of Auctions and Competitive Bidding, Econometrica, 50:5(1982), pp. 1089-1122.

[6] G. Riley and W. F. Samuelson, Optimal Auctions, American Economic Review, 71:3(1981), pp. 381-392.

[7] M. E. Oren and A.C. Williams, On Competitive Bidding, Operations Research, 23(1975), pp. 1072-1079.

[8] Dimitri P. Bertsekas, Dynamic Programming: Deterministic and Stochastic Models, Prentice-Hall, Englewood Cliffs, N.J., 1987.

[9] Edwin K. P. Chong and Stanislaw H. ŻaK, An Introduction to Optimization, Wiley, New York, 1996.

[10] Mokhtar S. Bazaraa And C. M. Shetty, Nonlinear Programming Theory and Algorithms, Wiley, New York, 1979.

[11] Gilbert Strang, Linear algebra and its applications, San Diego, Harcourt, Brace, Jovanovich, 1988. 